\title{
Addressing Mental IIlness Stigma, Implicit Bias, and Stereotypes in Medical School
}

\author{
Asia T. McCleary-Gaddy ${ }^{1}$ (D) Renay Scales $^{2}$ \\ Received: 4 February 2019 / Revised: 30 May 2019 / Accepted: 3 June 2019 /Published online: 13 June 2019 \\ (C) Academic Psychiatry 2019
}

"I regret that only now, in my last annual meeting speech, am I telling you about my own struggles. My fear of being judged negatively and the dark shadow of stigma nearly kept me from seeking help."

- 2018 Association of American Medical Colleges President and CEO Darrell Kirch, MD

Studies over the past five decades have revealed that physicians die from suicide more often than their non-physician counterparts do [1]. Between 2000 and 2014, 324 students (220 men, 104 women) died while in residency, with suicide being the leading cause for men [2]. The suicide rate among male physicians has been reported to be more than $40 \%$ higher than among men in the general population, whereas female physicians are more than $130 \%$ higher than among women in the general population [1]. Interestingly, the increased risk for suicide among physicians may begin during medical school [3]. An abundance of research reveals that medical students have a substantially lower mental quality of life than similarly aged individuals in the general population [1]. In a 2002 study of 194 medical students, $24 \%$ met the criteria on a rating scale indicating the presence of moderate to severe depressive symptoms [4]. Moreover, in a large multi-institutional study of the US medical students, researchers found approximately one out of every nine students had thoughts of suicide in the past year [1]. In other words, suicidal ideation among medical students $(11.2 \%)$ was higher than for individuals of similar

Asia T. McCleary-Gaddy

asia.mcclearygaddy@shu.edu

1 Hackensack Meridian School of Medicine at Seton Hall University, Nutley, NJ, USA

2 University of Kentucky College of Medicine, Lexington, KY, USA age in the general US population $(6.9 \%$ among $25-$ to $34-$ year-olds) [1].

In a 2002 study, the most frequently cited barriers to treatment were lack of time (48\%), lack of confidentiality (37\%), stigma (30\%), cost (28\%), fear of documentation on academic record (24\%), and fear of unwanted intervention (26\%) [3]. As a result, in 2002, the American Foundation for Suicide Prevention released a consensus statement identifying several key measures to address physician suicide [3]. These recommendations include healthcare provider annual anonymous screenings for mood disorders, substance abuse disorders, and/or suicidality: learning how to self-assess for depression and suicidality; non-discriminatory state re-licensing based on professional performance; free institution wellness programs, medical education curricula on physician depression, suicide and protective factors, and data collection on mental health prevalence and treatment services [3]. Although these recommended measures to address physician suicide are important, none of them directly address the third most cited barrier to treatment: stigma.

\section{Mental Illness Stigma}

Mental illness stigma refers to the devalued social identity one may possess due to the negative attribute of mental illness [5]. Mental illness stigma deters affected individuals from treatment by decreasing self-esteem, increasing feelings of shame, and limiting social opportunities [5]. For medical students, mental illness stigma may elicit perceptions of incompetence and create a stagnation in career trajectory in the competitive medical setting. Since mental illness is a concealable stigma; an identity people can choose to make known to others, many medical students may never reveal the status of their mental health, which may increase the risk of suicidal behaviors [4]. 
If the medical field aims to reduce stigmatization of mental illness and treatment, the fundamental causes of stigma need to be addressed. Initiatives that decrease mental illness stigma target the attitudes and behaviors that might be barriers to seeking care [5]. Historically, three main strategies were used to combat stigma: contact, education, and protest [5]. The current article takes a more nuanced approach and outlines three recommendations to address mental illness stigma in medical students and potentially decrease the future prevalence of physician suicide. First, the authors discuss the relationship between positive interpersonal contact with people with mental illness and its effect on implicit bias and prejudice. Secondly, the authors discuss the necessity of environmental vulnerability that encourages positive self-perceptions and reduces negative self-stereotypes of medical students. Lastly, the authors discuss advocacy at the organizational level to address structural stigma and perceptions of policies that limit opportunity.

\section{Positive Interpersonal Contact and Implicit Bias}

Stigma is reduced when individuals have contact with people with mental illness who are able to successfully and effectively function in society [5]. Direct contact with a successful individual who has mental illness helps change negative stereotypes and erroneous beliefs about mental illness by creating an opportunity in which non-stigmatized individuals can develop attitudes based on their own experience [6]. Researchers find this to be the most effective strategy in reducing mental illness stigma because it combines education with real-life experience versus negative stereotypes reinforced in society [7]. In a meta-analysis of interpersonal contact and the stigma of mental illness, researchers found that self-reported contact, job training, and laboratory studies in which contact with an individual with mental illness is manipulated all resulted in a reduction in stigmatizing perceptions of persons with mental illness [6]. In another meta-analysis, where contact was evaluated in the context of student or employee training in medical settings, researchers found contact with individuals with psychiatric disorders was associated with improved attitudes [8].

Therefore, opportunities for medical students and residents to meet physicians with mental illness may diminish stigma. The early exposure to successful healthcare practitioners with mental illness has the potential to moderate a student's expectation of discrimination or marginalization in medical settings.

An accessible opportunity for such intentional contact in undergraduate and graduate medical education is through colloquium series and Grand Rounds. The invitation of a physician with mental illness to these platforms communicates that the speaker is competent, evidence-based, and valued within the medical community. Consequently, it may also be an effective method in reaffirming a successful future for medical students who choose to disclose their mental illness and pursue treatment.

In addition to interpersonal contact with people with mental illness being a predictor of positive attitude change, it may also affect implicit bias [9]. Implicit bias is the attitudes or stereotypes that affect our understanding, actions, and decisions in an unconscious manner [10]. In a comparison study of people diagnosed with mental illness and people who were mentally healthy, both samples displayed implicit negative attitudes and beliefs about people who are mentally ill [11]. In an experiment examining the effect of positive counterstereotypic exposure (e.g., women leaders) on implicit bias, researchers found a significant decrease in negative implicit bias when exposed to examples counter to stereotypes [12]. Positive intergroup contact with physicians with mental illness can decrease implicit bias by changing the negative cognitive representations of people with mental illness and changing the negative evaluation of the group. Thus, medical student contact with physicians who have mental illness may work in two ways: altering both explicit and implicit attitudes and cognitions.

\section{Environmental Vulnerability and Self-Stereotyping}

Past research has shown that education related to mental illness stigma can improve attitudes toward people with mental illness [7]. For example, graduate students who enrolled in a course on mental illness showed more positive attitudes about people with mental illness than those who did not enroll in the course [7]. Education provides information that replaces myths about mental illness with facts and allows people to have more informed opinions about mental illness. Furthermore, education has also been shown to increase confidence in the effectiveness of treatment participation [5].

One major aspect of education is the learning environment. Medical student perceptions of the learning environment are an important determinant of educational attainment. Medical institutions can be stigmatizing due to competitiveness and a quest for perfection that may contribute to a fear of showing vulnerability. In a study measuring stereotypes of doctors and nurses by medical students, junior medical students considered doctors significantly more confident, arrogant, and detached than nurses [13]. In another study of perfectionism in medical students, maladaptive perfectionism defined as excessive evaluative concerns was significantly correlated with distress symptoms and was predictive of depression and hopelessness [14]. Therefore, although education has been shown to be a successful method to combat stigma in most settings, there is a unique set of characteristics that need to be addressed 
in the medical school setting. Learning in an environment in which stereotyping is pervasive may diminish self-esteem and confidence to share mental health issues. It may also increase feelings of imposter syndrome and other self-perceptions that create barriers to growth.

In order for medical students with mental illness to disclose their mental health status and seek treatment, an environment of vulnerability needs to be established within the curriculum. One method shown to increase vulnerability and create a conducive atmosphere to learn is self-reflection. Vulnerability can be encouraged through self-reflection exercises that help students build self-awareness and competence [15]. Selfreflection helps students examine their own attitudes and beliefs relating to prior experiences in medicine (reflection on action) and the current practice of medicine (reflection in action) [16]. Self-reflection exercises designate class time and graded assignments to exploring vulnerable emotions. Subsequently, this confers importance and value to vulnerability in medical education. As a result, medical students who disclose their mental illness through reflective practices will feel valued rather than shamed because it is taught that this vulnerability is an important aspect of medical education and professional identity. Past research suggests that the effects of education may not endure over time [5]. However, making self-reflection and education a consistent part of the curriculum may increase the effects of education.

\section{Organizational Advocacy and Structural Change}

Stigma as a social cognitive construct is only one of numerous stigma-related dynamics that act as a barrier to accessing mental health care [17]. Structural stigma is defined as the economic and political pressures on a culture that produce social and institutional policies that limit opportunities for the stigmatized group [5]. Structural stigma includes institutional policies that intentionally constrain the opportunities of people with mental illness but also the unintentional policies that are not intended to be a detriment but whose consequences impede the options of people with mental illness [17]. A reported concern of both people with mental illness and medical professionals is threats to confidentiality [3]. Many people may avoid mental health services because they fear that confidential information about their psychiatric history may become exposed. Medical students with mental illness have the added concern of this being documented on their academic record and potentially decreasing their likelihood of preferred specialty placement, academic awards, and physician licensing [3]. One example is how nearly two thirds of state medical boards' licensure applications inquire about whether the applicant has ever had a mental health condition that could possibly impair the ability to practice medicine rather than inquiring about current (within a time period of 12 months or less) impairment from a medical condition or mental health condition [18].

Policies that increase mental illness stigma may be reduced when groups challenge the way the stigma is represented. The Mental Health Association and the Substance Abuse and Mental Health Services Administration have both successfully created anti-stigma campaigns to change the culture and perception of how people who suffer from mental illness, including substance use disorders, are viewed by society [5]. It may be effective for organizations such as the American Association for Medical Colleges, the Federation of State Medical Boards, and the Joint Commission on the Accreditation of Healthcare Organizations to do the same. Using a strategic plan of targeted population messaging, medical institutions can create a unique anti-stigma campaign of inclusion and assistance to medical students with mental illness. This would reinforce a national climate that normalizes the explicit conversation of mental illness in medical professionals and openly provides the tools to address it. Moreover, conversations addressing economical and structural barriers would convey that these are priorities in medical education and begin to change the culture in medicine.

\section{Changing the Culture}

Research suggests that mental illness stigma may impede medical students from seeking mental health services [3]. However, it can be argued that mental illness stigma in medical students is not only a barrier to care access but may also increase the likelihood of future physician suicide. When we examine how mental illness stigmatization among medical students can be understood in terms of implicit bias, stereotypes, and unequal opportunity, it is evident why addressing mental health stigma is key to creating a culture of equity, inclusion, and healthy well-being for medical students [5]. One benefit to the multi-faceted nature of mental illness stigma is that it can be addressed in a multitude of ways. Although systemic change may take an extended amount of time until the effects of change are acknowledged by its beneficiaries, the recommendations described above offer interventions at the individual, institutional, and organizational level. Fundamental in each of these strategies is an implicit incentive for medical students to reveal a concealed identity of mental illness because anticipation of devaluation by the medical community is eliminated. Consequently, this may increase medical students' treatment-seeking behaviors. In conclusion, addressing mental illness stigma early at the medical school level may be a promising avenue for decreasing physician suicide later at the medical professional level. Moreover, it is an unexplored path for advancing the culture of medicine. 


\section{Compliance with Ethical Standards}

Disclosure On behalf of all authors, the corresponding author states that there is no conflict of interest.

\section{References}

1. Dyrbye LN, Thomas MR, Massie FS, Power DV, Eacker A, Harper W, et al. Burnout and suicidal ideation among U.S. medical students. Ann Intern Med. 2008;149:334-41. https://doi.org/10.7326/ 0003-4819-149-5-200809020-00008.

2. Yaghmour NA, Brigham TP, Richter T, Miller RS, Philibert I, Baldwin DWC Jr, et al. Causes of death of residents in ACGMEaccredited programs 2000 through 2014: implications for the learning environment. Acad Med. 2017;92(7):976-83.

3. Hampton T. Experts address risk of physician suicide. JAMA. 2005;294(10):1189-91. https://doi.org/10.1001/jama.294.10.1189.

4. Givens JL, Tjia J. Depressed medical students' use of mental health services and barriers to use. Acad Med. 2002;77:918-21.

5. Corrigan P. How stigma interferes with mental health care. Am Psychol. 2004;59:614-25. https://doi.org/10.1037/0003-066X.59. 7.614.

6. Couture S, Penn DL. Interpersonal contact and the stigma of mental illness: a review of the literature. J Ment Health. 2003;12:291-305.

7. Corrigan PW, River LP, Lundin RK, Penn DL, Uphoff-Wasowski $\mathrm{K}$, Campion J, et al. Three strategies for changing attributions about severe mental illness. Schizophr Bull. 2001;27:187-95.

8. Kolodziej ME, Johnson BT. Interpersonal contact and acceptance of persons with psychiatric disorders: a research synthesis. J Consult Clin Psychol. 1996;64:1387-96. https://doi.org/10.1037/ 0022-006X.64.6.1387.

9. Stathi S, Tsantila K, Crisp R. Imagining intergroup contact can combat mental health stigma by reducing anxiety, avoidance and negative stereotyping. J Soc Psychol. 2012;152(6):746-57. https:// doi.org/10.1080/00224545.2012.697080.
10. Devine PG, Forscher PS, Austin AJ, Cox WT. Long-term reduction in implicit race bias: a prejudice habit-breaking intervention. J Exp Soc Psychol. 2012;48(6):1267-78.

11. Teachman B, Wilson J, Komarovskaya I. Implicit and explicit stigma of mental illness in diagnosed and healthy samples. J Soc Clin Psychol. 2006;25:75-95.

12. Dasgupta N, Asgari S. Seeing is believing: exposure to counterstereotypic women leaders and its effect on the malleability of automatic gender stereotyping. J Exp Soc Psychol. 2004;40: $642-58$.

13. Rudland JR, Mires GJ. Characteristics of doctors and nurses as perceived by students entering medical school: implications for shared teaching. Med Educ. 2005;39(5):448-55. https://doi.org/ 10.1111/j.1365-2929.2005.02108.x.

14. Enns MW, Cox BJ, Sareen J, Freeman P. Adaptive and maladaptive perfectionism in medical students: a longitudinal investigation. Med Educ. 2001;35:1034-42. https://doi.org/10.1046/j.13652923.2001.01044.x.

15. Stephens M, Reamy B, Anderson D, Olsen C, Hemmer P, Durning $\mathrm{S}$, et al. Writing, self-reflection, and medical school performance: the human context of health care. Mil Med. 2012;177:26-30.

16. Epstein RM. Mindful practice. JAMA. 1999;282:833-9.

17. Corrigan PW, Markowitz FE, Watson AC. Structural levels of mental illness stigma and discrimination. Schizophr Bull. 2004;30(3): 481-49.

18. Dyrbye LN, West CP, Sinsky CA, Goeders LE, Satele DV, Shanafelt TD. Medical licensure questions and physician reluctance to seek care for mental health conditions. Mayo Clin Proc. 2017;92:1486-93.

Publisher's Note Springer Nature remains neutral with regard to jurisdictional claims in published maps and institutional affiliations. 